\title{
A MARTINGALE INEQUALITY RELATED TO EXPONENTIAL SQUARE INTEGRABILITY
}

\author{
JILL PIPHER
}

(Communicated by J. Marshall Ash)

\begin{abstract}
We present an inequality for dyadic martingales (together with its continuous analog for functions on $\mathbb{R}^{n}$ ) which is shown to be equivalent to a result of Chang-Wilson-Wolff on exponential square integrability. The analog of this weighted inequality for double dyadic martingales is also proven. Finally, we discuss a possible connection between these inequalities and a theorem of Garnett.
\end{abstract}

In this note, we present an inequality for dyadic martingales (together with its continuous analog for functions on $\mathbb{R}^{n}$ ) which is shown to be equivalent to a result of Chang-Wilson-Wolff [1] on exponential square integrability. The proof of our martingale inequality is not hard; it is a consequence of a lemma in [1], which is originally due to Wald (see $[9$, p. 160]). There are several interesting features of this inequality between a martingale and its square functions. In the first place, it is extremely simple to state. Second, significant inequalities relating functions and their square functions can, in one direction, be difficult to establish. For examples, see Wilson [7, 8]. Third, the lack of homogeneity crucial in this lemma is reflected here without exponentiation. Finally, there seems to be an interesting connection between the inequality and a theorem of Garnett [5] which we discuss in $\S 2$.

\section{An INEQUality}

Let $f_{N}=\sum_{0}^{N} d_{n}$ be a dyadic martingale on $[0,1]$, i.e., if $\mathscr{F}_{n}$ is the $\sigma$-field generated by the dyadic subintervals of length $2^{-n}$ then $E\left(f_{N} \mid \mathscr{F}_{n}\right)=f_{n}$ and $\int_{0}^{1} d_{n}=0$. Assume that $f_{0}=d_{0}=0$. The square function of $f_{N}$ is given by $S_{N}^{2} f=\sum_{0}^{N} d_{n}^{2}$. It is well known that

$$
C_{p}^{-1}\left\|f_{N}\right\|_{p} \leq\left\|S_{N} f\right\|_{p} \leq B_{p}\left\|f_{N}\right\|_{p}
$$

and the issue taken up in [1] was the determination of the sharp dependence on $p$ of $C_{p}$ and $B_{p}$. We shall concentrate on the inequality

$$
\left\|f_{N}\right\|_{p} \leq C_{p}\left\|S_{N} f\right\|_{p},
$$

Received by the editors April 25, 1990 and, in revised form, October 15, 1991.

1991 Mathematics Subject Classification. Primary 42B25; Secondary 42C10.

Supported in part by an NFS postdoctoral research fellowship and a Sloan Fellowship. 
where the cancellation, and not merely the size, of the $\left\{d_{n}\right\}$ is critical. It was shown in [1] that $c_{p} \sim \sqrt{p}$ as $p \rightarrow+\infty$ as a consequence of a stopping time argument using the result

$$
\int_{0}^{1} e^{f_{N}-S_{N}^{2} f / 2} d x \leq 1
$$

Inequality (1.2) is referred to as Rubin's lemma and can be rescaled to show that $\left|f_{N}\right|$ is exponentially square integrable whenever $S f_{N} \in L^{\infty}([0,1])$. The continuous analogue of this fact for functions on $\mathbb{R}^{n}$ is the following inequality, reminiscent of the defining condition for BMO. If $f_{Q}=\frac{1}{|Q|} \int_{Q} f d x$ denotes the average of $f$ over a cube $Q$ in $\mathbb{R}^{n}$, then there exists a $\delta>0$ such that

$$
\sup _{Q \subseteq \mathbb{R}^{n-1}}\left\{\frac{1}{|Q|} \int_{Q} e^{\delta\left|f-f_{Q}\right|^{2} /\|S f\|_{\infty}^{2}}\right\}<+\infty
$$

where

$$
S^{2} f\left(x_{0}\right)=\int_{\left\{(x, y):\left|x-x_{0}\right|<y\right\}} y^{2-n}|\nabla u(x, y)|^{2} d x d y
$$

and $u$ is the harmonic extension of $f$ to $\mathbb{R}_{+}^{n}$.

Let $M w$ denote the Hardy-Littlewood maximal function of $w$ relative to the interval $[0,1]$. For dyadic martingales on $[0,1]$ we have the following result.

Theorem 1.4. There is a constant $C$ such that

$$
\int_{0}^{1}\left|f_{N}\right| w d x \leq C\left\{\int_{0} S_{N}^{2} f w d x+\int_{0}^{1} M w d x\right\}
$$

Corollary 1.6. If $\left\|S f_{N}\right\|_{L^{\infty}}<+\infty$, then there exists an $\alpha>0$ such that

$$
\int_{0}^{1} e^{\alpha\left|f_{n}\right|^{2} /\|S f\|_{\infty}^{2}} d x \leq C .
$$

Proof of Corollary 1.6 (assuming Theorem 1.4). The corollary follows by rescaling (1.5). Multiplying $f_{N}$ by $\varepsilon$ and $w$ by $\varepsilon^{-1}$ gives

$$
\int_{0}^{1}\left|f_{N}\right| w d x \leq \frac{C}{\varepsilon}\left\|S^{2} f_{N}\right\|_{\infty} \int_{0}^{1} w d x+C \varepsilon \int_{0}^{1} M w d x .
$$

To test the $L^{p}$ norm of $\left|f_{N}\right|$, let $w \geq 0$ satisfy $\|w\|_{L p^{\prime}} \leq 1, \frac{1}{p}+\frac{1}{p^{\prime}}=1$ and observe that

$$
\int_{0}^{1} M w d x \leq\|M w\|_{L p^{\prime}} \leq \frac{A}{p^{\prime}-1}\|w\|_{L^{p^{\prime}}} \leq A p
$$

for $1<p^{\prime}<2$. Hence,

$$
\left\|f_{N}\right\|_{L^{p}} \leq\left\|S_{N} f\right\|_{\infty}^{2} \frac{C}{\varepsilon}+C A \varepsilon p
$$

and choosing $\varepsilon=\left\|S_{N} f\right\|_{\infty} / \sqrt{p}$ yields $\left\|f_{N}\right\|_{L^{p}} \leq C^{\prime}\left\|S f_{N}\right\|_{\infty} \sqrt{p}$. Thus if $\alpha$ is sufficiently small,

$$
\begin{aligned}
\int_{0}^{1} e^{\alpha\left|f_{N}\right|^{2} /\left\|S f_{N}\right\|_{\infty}^{2}} & \leq \sum_{p=0}^{\infty} \frac{\alpha^{p}}{p !} \int_{0}^{1}\left(\frac{\left|f_{N}\right|}{\left\|S f_{N}\right\|_{\infty}}\right)^{2 p} d x \\
& \leq \sum_{p=0}^{\infty} \frac{\alpha^{p}}{p !}\left(C^{\prime}\right)^{2 p} \cdot p^{p}<+\infty .
\end{aligned}
$$


Using a more general form of Corollary 1.5 , namely that for any dyadic $Q \subseteq[0,1]$ of length $2^{-n}$,

$$
\int_{Q} \exp \left\{a\left|\sum_{p=n}^{N} d_{p}\right| /\left\|\sum_{p=n}^{N} d_{p}\right\|_{\infty}^{2}\right\} \leq C|Q|
$$

we see that

$$
\left|\left\{x \in Q:\left|f_{N}\right|>\lambda\right\}\right| \leq C e^{-\alpha \lambda^{2} /\|S f\|_{\infty}^{2}}|Q|
$$

and a stopping time argument yields

$$
\left|\left\{x: f^{*}>2 \lambda, S f<\varepsilon \lambda\right\}\right| \leq C \exp \left(-\alpha \frac{(1-\varepsilon)^{2} \lambda^{2}}{\varepsilon^{2} \lambda^{2}}\right) \cdot\left|\left\{f^{*}>\lambda\right\}\right|,
$$

where $f^{*}(x)=\sup _{n}\left|\sum_{p=0}^{n} d_{p}(x)\right|$.

Proof of Theorem 1.4. Let $w$ be a nonnegative function on $[0,1]$ with $\|w\|_{L \log L}=\int_{0}^{1} w\left(1+\log ^{+} w\right) d x$. Let $g^{+}$denote $g \chi_{\{g \geq 0\}}$. Then

$$
\left|f_{N}\right| \leq\left(f_{N}-S^{2} f_{N} / 2\right)^{+}+\left(-f_{N}-S^{2} f_{N} / 2\right)^{+}+S^{2} f_{N} / 2 \text {. }
$$

Hence to prove inequality (1.5) it suffices to consider the two integrals $\int_{0}^{1}\left(f_{N}-S^{2} f_{N} / 2\right)^{+} w d x$ and $\int_{0}^{1}\left(-f_{N}-S^{2} f_{N} / 2\right)^{+} w d x$. We now observe that $\left(f_{N}-S^{2} f_{N} / 2\right)^{+}$belongs to the class $e^{L}$ since

$$
\int_{0}^{1} e^{\left(f_{N}-S^{2} f_{N} / 2\right)^{+}} d x=\int_{\left\{f_{N}>S^{2} f_{N} / 2\right\}} e^{f_{N}-S^{2} f_{N} / 2} d x+\left|\left\{f_{N}<S^{2} f_{N} / 2\right\}\right| \leq 2,
$$

since by inequality (1.2) each term is bounded by one. Now (1.2) applies as well to the expression $\exp \left(-F_{N}-S^{2} f_{N} / 2\right)^{+}$and so the term $\left(-f_{N}-S^{2} f_{N} / 2\right)^{+}$ is in $e^{L}$ with the same bound. The class $L \log L$ is dual to $e^{L}$ and thus

$$
\int_{0}\left|f_{N}\right| w \leq \frac{1}{2} \int_{0}^{1} S^{2} f_{N} w+\|w\|_{L \log L} \leq \frac{1}{2} \int_{0}^{1} S^{2} f_{N} w+\int_{0}^{1} M w .
$$

Remarks. (1) Inequality (1.5) has a continuous analog, whose proof is a reduction to the martingale situation using the arguments of [1],

$$
\int_{Q}\left|f(x)-f_{Q}\right| w(x) d x \leq C \int_{Q} S^{2} f(x) w(x) d x+C \int_{Q} M w(x) d x .
$$

This inequality, in turn, implies (1.3).

(2) Two parameter versions of Theorem 1.4 can be shown by iteration. If $\left\{f_{n, m}\right\}$ is a doubly indexed sequence such that for each fixed $n,\left\{f_{n, m}\right\}$ is a dyadic martingale relative to $n$ (and similarly for each fixed $m$ ) then it is called a double dyadic martingale. For $d_{n, m}=f_{n, m}-f_{n-1, m}-f_{n, m-1}+f_{n-1, m-1}$, the square function of $f_{N, N}$ is defined as

$$
S^{2} f_{N, N}=\sum_{n=0}^{N} \sum_{m=0}^{N} d_{n, m}^{2} .
$$

We may now define (see [6]) a "partial" square function of $f_{N, N}$ by setting

$$
S_{1}^{2} f_{N, N}\left(x_{1}, x_{2}\right)=\sum_{n=0}^{N}\left(\sum_{m=0}^{N} d_{n, m}\right)^{2}\left(x_{1}, x_{2}\right) \text {. }
$$


The proof of Rubin's lemma yields

$$
\int \exp \left(f_{N, N}-\frac{1}{2} \sum_{n=0}^{N}\left(\sum_{m=0}^{N} d_{n, m}\left(x_{1}, x_{2}\right)\right)^{2}\right) d x_{1} \leq 1
$$

and Lemma 2.2 of [6] yields

$$
\int \exp \left(\sqrt{1+S_{1}^{2} f_{N, N}\left(x_{1}, x_{2}\right)}-S^{2} f_{N, N}\left(x_{1}, x_{2}\right)\right) d x_{2} \leq e .
$$

Then, (1.10) and (1.11) combine to prove

$$
\begin{aligned}
\int_{0}^{1} \int_{0}^{1}\left|f_{N, N}\left(x_{1}, x_{2}\right)\right| w\left(x_{1}, x_{2}\right) d x_{1} d x_{2} \\
\quad \leq C \int_{0}^{1} \int_{0}^{1} S^{2} f_{N, N}\left(x_{1}, x_{2}\right) w\left(x_{1}, x_{2}\right) d x_{1} d x_{2} \\
\quad+C \int_{0}^{1} \int_{0}^{1} M_{1} M_{2} w\left(x_{1}, x_{2}\right) d x_{1} d x_{2},
\end{aligned}
$$

where $M_{i}, i=1,2$, is the Hardy-Littlewood maximal operator in the variable $x_{i}$. We do not know if inequality $(1.12)$ is valid when $\left(M_{1} M_{2} w\right)$ is replaced by the rectangular maximal operator

$$
M_{S} w(\bar{x})=\sup _{R \ni \bar{x}}\left(\frac{1}{|R|} \int_{R} w(z) d z\right)
$$

where the supremum is taken over rectangles parallel to the axes.

\section{REMARKS}

We wish to point out what seems to be an interesting connection between inequality (1.9) and a theorem of Garnett [5]. We shall consider Dahlberg's formulation [3] as he gave the best constants and it is this dependence on the constants which is important in the context. It would be desirable to replace the "philosophical" discussion which follows by something explicit. This would entail a new proof (without a reduction to the martingale situation) of (1.9), which should be of general interest.

The issue taken up in [3] and [5] is the relationship between BMO functions on $\mathbb{R}^{n}$ and Carleson measure properties of their extensions to the upper half space $\mathbb{R}_{+}^{n+1}$. It is well known [4] that if $f \in \operatorname{BMO}\left(\mathbb{R}^{n}, d x\right)$, then

$$
\sup _{Q \text { a cube }}\left\{\int_{x \in Q} \int_{y=0}^{l(Q)} y|\nabla u(x, y)|^{2} \frac{d x d y}{|Q|}\right\} \leq C
$$

(where $l(Q)=$ length of the cube $Q$ ). It would be preferable for $|\nabla u(x, y)| d x d y$ to satisfy a Carleson measure condition, rather than the quadratic expression in (2.1) above, but this is false. One may still ask if there exists some extension $\varphi(x, y)$ to $\mathbb{R}_{+}^{n}$ of $f(x)$ such that $|\nabla \varphi| d x d y$ is Carleson and this is the case.

Theorem 2.2 (Dahlberg [3]). Suppose $u$ is harmonic in a bounded Lipschitz domain $D \subseteq \mathbb{R}^{n}, n \geq 2$. Then for every $\varepsilon>0$ there is a function $\varphi$ such that 
$|u-\varphi| \leq \varepsilon$ in $D$ and for all $P \in \partial D$ we have that

$$
\int_{\beta(r)}|\nabla \varphi| d X \leq \frac{C}{\varepsilon} \int_{\beta\left(c_{r}\right)}|\nabla u|^{2} \operatorname{dist}(X, \partial D) d X+C \varepsilon r^{n-1},
$$

where $\beta(r)=\{x \in D:|x-P| \leq r\}$ and $C$ depends on $D$.

For our purposes, let $D=[0,1] \times[0,1]$ and suppose $f(x)$ is a given, say $L^{2}$, function on $[0,1]$ and let $u$ be the harmonic extension of $f$ to $\mathbb{R}_{+}^{2}$. Let $Q$ be a fixed interval in [0,1] of length $h$. For $\varepsilon>0$ given, let $\varphi(x, y)$ be the function given by Dahlberg's theorem above. Fix an $x_{0} \in Q$. We see that, for any $x \in Q$,

$$
\varphi\left(x_{0}, 0\right)-\varphi(x, h)=-\int_{l} \nabla \varphi \cdot d \vec{r}
$$

where $l$ is the line segment joining $\left(x_{0}, 0\right)$ and $(x, h)$. If we average both sides of (2.4) over $Q$ and change variables, we obtain

$$
\left|\varphi\left(x_{0}, 0\right)-f_{Q} \varphi(x, h) d x\right| \leq \int_{\Gamma\left(x_{0}\right)}\left|\nabla \varphi\left(x^{\prime}, y^{\prime}\right)\right| d x^{\prime} \frac{d y^{\prime}}{y^{\prime}}
$$

where $f_{Q} \cdots d x$ denotes $\int_{Q} \cdots \frac{d x}{|Q|}$ and $\Gamma\left(x_{0}\right)=\left\{\left(x^{\prime}, y^{\prime}\right):\left|x^{\prime}-x_{0}\right|<y^{\prime}\right\}$. Let $c_{Q}$ be the constant $f_{Q} u(x, h) d x$. Then,

$$
\begin{aligned}
\left|f(x)-c_{Q}\right| \leq & |f(x)-\varphi(x, 0)|+\left|\varphi(x, 0)-f_{Q} \varphi(x, h) d x\right| \\
& +\left|\int_{Q}[u(x, h)-\varphi(x, h)] d x\right| \\
\leq & 2 \varepsilon+\int_{\Gamma(x)}\left|\nabla \varphi\left(x^{\prime}, y^{\prime}\right)\right| d x^{\prime} \frac{d y^{\prime}}{y^{\prime}},
\end{aligned}
$$

by (2.5) and the properties of $\varphi$ given by Theorem 2.2. Consider now the left-hand side of the inequality; we wish to prove

$$
\int_{Q}\left|f-c_{Q}\right| w d x \leq C\left\{\int_{Q} S^{2}(f) w d x+\int_{Q} M w\right\} .
$$

Inserting the above estimate for $\left|f-c_{Q}\right|$, we see that

$$
\int_{Q}\left|f-c_{Q}\right| w d x \leq 2 \varepsilon \int_{Q} w d x+\int_{Q} \int_{\Gamma(x)}\left|\nabla \varphi\left(x^{\prime}, y^{\prime}\right)\right| d x^{\prime} \frac{d y^{\prime}}{y^{\prime}} w d x .
$$

In Theorem 2.2, the $L^{1}$ square function of $\varphi, \int_{\Gamma}|\nabla \varphi| d x^{\prime} d y^{\prime} / y^{\prime}$, is not dominated pointwise by $\int_{\Gamma}|\nabla u|^{2} d x^{\prime} d y^{\prime}$. (If it were, (2.6) would follow immediately.) Viewing (2.3) as an inequality for square functions, it would appear that (2.6) could be a weighted version of (2.3). Therefore, the maximal function of $w$ appears since no special assumptions on $f w$ are made (e.g., $w \in A_{\infty}(d x)$ [2]). It should therefore be of interest to consider weighted versions of (2.3), under weak, or no, assumptions on $w$. 


\section{REFERENCES}

1. S.-Y. A. Chang, J. M. Wilson, and T. H. Wolff, Some weighted norm inequalities for the Schrödinger operator, Comment. Math. Helv. 60 (1985), 217-246.

2. R Coifman and C. Fefferman, Weighted norm inequalities for maximal functions and singular integrals, Studia Math. 51 (1974), 241-250.

3. B. E. J. Dahlberg, Approximation of harmonic functions, Ann. Inst. Fourier (Grenoble) 30 (1980), 97-107.

4. C. Fefferman and E. Stein, $H^{p}$ spaces of several variables, Acta Math. 129 (1972), 137-192.

5. J. Garnett, Two constructions in BMO, Proc. Sympos. Pure Math., vol. 35, Amer. Math. Soc., Providence, RI, 1979, pp. 295-301.

6. J. Pipher, Bounded double square functions, Ann. Inst. Fourier (Grenoble) 36 (1986), 69-82.

7. J. M. Wilson, Weighted inequalities for the dyadic square function without dyadic $A_{\infty}$, Duke Math. J. 55 (1987), 19-50.

8. _ _ A sharp inequality for the square function, Duke Math. J. 55 (1987), 879-888.

9. A. Wald, Sequential analysis, Wiley, New York, 1974.

Department of Mathematics, University of Chicago, Chicago, Illinois 60637 02906

Current address: Department of Mathematics, Brown University, Providence, Rhode Island

E-mail address: jpipher@gauss.math.brown.edu 